Original Paper http://ajol.info/index.php/ijbcs http://indexmedicus.afro.who.int

\title{
Performances de production des cobayes (Cavia porcellus L.) en fonction du niveau de protéines alimentaires
}

\author{
G.T. ZOUGOU ${ }^{1,2}$, F. TENDONKENG ${ }^{1}$, E. MIEGOUE ${ }^{1}$, M.N. NOUMBISSI ${ }^{1}$, \\ A.V. MBOKO ${ }^{2}$, F.E.N. MATUMUINI ${ }^{2}$, B. BOUKILA ${ }^{2}$ et T. E. PAMO ${ }^{1 *}$ \\ ${ }^{I}$ Laboratoire de Nutrition Animale, Département des Productions Animales, FASA, Université de Dschang, \\ B.P. 222 Dschang, Cameroun. \\ ${ }^{2}$ Institut National Supérieur d'Agronomie et de Biotechnologies (INSAB), Université des Sciences et \\ Techniques de Masuku, B.P. 941 Franceville, Gabon. \\ *Auteur correspondant ; E-mail :ftendonkeng@yahoo.fr/f.tendonkeng@univ-dschang.org; \\ Tél : +(237) 699545432.
}

\section{RESUME}

Au Cameroun, l'élevage des cobayes se pratique encore dans les cuisines et à même le sol et leur alimentation est essentiellement à base des déchets de cuisine. Le souci d'intensifier la production nécessite la détermination des besoins réels du cobaye afin d'asseoir de façon rationnelle sa table de rationnement. L'objectif de ce travail est donc de déterminer les taux optimums de protéines dans son alimentation en fonction de son état physiologique. Pour cet essai, 75 femelles ont été préalablement mises en croisement avec 12 mâles pendant une période de 31 jours selon un dispositif factoriel de trois lots comptant 25 femelles chacun. Chaque lot recevait une ration contenant $18 \%, 20 \%$ ou $22 \%$ de protéine brute (PB). Après le sevrage, 54 jeunes cobayes ont été constitués en 3 lots homogènes de 18 animaux ( 9 mâles et 9 femelles) chacun. Chaque lot recevait une ration contenant $14 \%, 16 \%$ ou $18 \%$ de PB. Aucune différence significative $(P>0,05)$ n'a été observée entre la taille de la portée et les poids des femelles allaitantes de la mise-bas au sevrage. Le poids à la naissance des cochonnets des femelles recevant la ration contenant $22 \%$ de PB (RG22) a été significativement $(\mathrm{p}<0,05)$ plus élevé que ceux issus des femelles des autres lots. Par ailleurs, les taux de fertilité $(96 \%)$, de fécondité $(184 \%)$, de fécondité nette $(184 \%)$, de viabilité à la naissance $(100 \%)$ et de viabilité au sevrage $(97,83 \%)$ les plus élevés ont été également enregistrés chez ces femelles soumises à la ration contenant $22 \%$ de PB (RG22). Le gain total, le gain moyen quotidien (GMQ) et l'indice de consommation (IC), respectivement $60,20 \mathrm{~g} ; 2,15 \mathrm{~g} / \mathrm{j}$ et 1,67 chez les mâles et $52,72 \mathrm{~g} ; 1,88 \mathrm{~g} / \mathrm{j}$ et 2,63 chez les femelles, ont été meilleurs chez les animaux nourris à la ration contenant $16 \%$ de PB (RC16). Tous ces paramètres ont été significativement $(\mathrm{P}<0,05)$ meilleurs chez les mâles comparés à ceux des femelles. Il en ressort que les rations contenant $22 \%$ (RG22) et 16\% (RC16) de PB, conviennent respectivement aux cobayes en gestation et en croissance à l'Ouest Cameroun.

(C) 2017 International Formulae Group. All rights reserved.

Mots clés: Cobayes, protéines alimentaires, performances de reproduction, performances de croissance, Ouest-Cameroun. 


\title{
Production performance of guinea pigs (Cavia porcellus L.) in function of dietary protein level
}

\begin{abstract}
In Cameroon, guinea pigs raising is still practiced in kitchens and on the ground and their food is essentially based on cooking waste. The desire to intensify production requires the determination of the actual needs of the guinea pig in order to rationally establish its rationing table. The objective of this work is to determine the optimum levels of protein in its diet as a function of its physiological state. For this work, 75 females were previously crossed with 12 males for a period of 31 days according to a factorial design of three groups with 25 females each. Each group received a ration containing 14\%, 16\% or 18\% of PB. No significant difference $(\mathrm{P}>0.05)$ was observed between litter size and lactating female weights from farrowing to weaning. The birth weight of the female pigs of the group PB22 $(22 \% \mathrm{~PB})$ was significantly $(\mathrm{p}<0.05)$ higher than that of the females of the other groups. In addition, fertility rates (96\%), fertility (184\%), net fertility (184\%), viability at birth $(100 \%)$ and viability at weaning $(97.83 \%)$ were also recorded in these females of group PB22. The total gain, the average daily gain (GMQ) and the consumption index (IC), respectively $60.20 \mathrm{~g} ; 2.15 \mathrm{~g} / \mathrm{j}$ and $1.67 \mathrm{in}$ males and $52.72 \mathrm{~g} ; 1.88 \mathrm{~g} / \mathrm{j}$ and 2.63 in females were better in the animals of group PB16. All of these parameters were significantly $(\mathrm{P}<0.05)$ improved in males compared with females. It appears that rations containing $22 \%(\mathrm{RG} 22)$ and $16 \%(\mathrm{RC} 16)$ of $\mathrm{PB}$, are suitable for guinea-pigs in pregnancy and growing in the highlands of western Cameroon.
\end{abstract}

(C) 2017 International Formulae Group. All rights reserved.

Keywords: Guinea pigs, dietary protein, reproductive performance, growth performance, West Cameroon.

\section{INTRODUCTION}

La demande de plus en plus croissante en protéines d'origine animale des populations locales, conduit à une pression accrue sur la faune sauvage; ce qui constitue aujourd'hui une sérieuse menace pour la survie de ces espèces animales (Fantondji et Soro, 2004 ; Awono et al., 2005). Pour assurer la préservation de ces ressources animales sauvages tout en satisfaisant les besoins en protéines d'origine animale, l'élevage non conventionnel en général et la caviaculture en particulier semble être une alternative. Elle constitue la forme de préservation ex - situ des espèces fauniques et une source non négligeable de protéines (Fantondji et Soro, 2004). Nkidiaka (2004) et Pamo et al. (2005) soulignent que la caviaculture semble être l'une des solutions durables pour répondre à ces besoins en protéines. En outre, cet élevage constitue une source importante de revenus pour des populations concernées (Fotsa et al.,
2007). Cependant, cet élevage est généralement pratiqué par les femmes et les enfants dans un système extensif (Niba et al., 2012). Les animaux sont élevés dans les cuisines et à même le sol. Leur alimentation est essentiellement à base des déchets de cuisine. Tout ceci ne permet pas à l'animal d'extérioriser son potentiel génétique (Niba et al., 2004b). A la lumière de tout ce qui précède, l'amélioration de la productivité du cochon d'inde s'avère indispensable. En élevage en général et en caviaculture en particulier, l'alimentation joue un rôle de choix (Tchoumboué, 2001 ; Kouakou et al., 2012; Bindelle et Picron, 2013). L'intensification de la production caviacole passe surtout par l'amélioration de l'alimentation (Bindelle et Picron, 2013). Il est donc nécessaire de déterminer les besoins réels du cobaye afin d'asseoir de façon rationnelle la table de rationnement pour cette espèce. Parmi les macronutriments, les 
protéines constituent le principal facteur limitant (Kondombo et al., 2003). Une alimentation contenant des quantités optimales de protéines améliorerait les performances de croissance et de reproduction des cobayes (Fonteh et al., 2005 ; Bindelle et Picron, 2013; Noumbissi et al., 2014; Miégoué, 2016). De meilleures viabilités sont observées chez les cobayes recevant une alimentation riche en protéines (Kouakou, 2016; Miégoué, 2016; Noumbissi, 2016). Compte tenu du coût élevé des compléments protéiques couramment utilisés en alimentation animale, il apparaît que l'apport optimum des protéines alimentaires dans la ration des cobayes pourrait réduire leur coût de production sans nuire à leur performance (Kouakou et al., 2010). Ainsi, nous avons étudié l'effet du niveau de protéines alimentaires sur les performances de reproduction et de croissance des cobayes à l'Ouest-Cameroun.

\section{MATERIEL ET METHODES}

\section{Site expérimental}

L'étude a été conduite entre avril 2015 et février 2016 à la Ferme d'Application et de Recherche (FAR) de l'Université de Dschang, située dans les hautes terres de l'OuestCameroun à une altitude de $1410 \mathrm{~m}$, à la longitude Est de $10^{\circ} 26^{\prime}$ et la latitude Nord de $5^{\circ} 26^{\prime}$. Cette région reçoit entre 1500 et 2000 $\mathrm{mm}$ de pluie par an, avec une température allant de $10^{\circ}$ à $25^{\circ} \mathrm{C}$. Le climat est équatorial de type camerounien d'altitude avec une courte saison sèche allant de mi-novembre à mi-mars et une longue saison pluvieuse de mimars à mi-novembre.

\section{Matériel animal et logement}

Quatre-vingt et sept (87) cobayes adultes de race anglaise $(418,92 \pm 46,75 \mathrm{~g})$ constitués de 75 femelles et 9 mâles âgés de 5 mois ont été utilisés pour cet essai. Les femelles ont été divisées en 3 lots de 25 animaux chacun, chaque lot était subdivisé en 2 sous-lots de 12 et 13 animaux repartis dans des loges mesurant 1,5 m de longueur, $0,8 \mathrm{~m}$ de largeur et $0,6 \mathrm{~m}$ de hauteur chacune et délimitées par des contreplaqués. Au sevrage, 54 jeunes cobayes issus de ces reproductrices ont été répartis en 3 lots homogènes de 18 animaux ( 9 mâles et 9 femelles) chacun. Ces jeunes animaux ont été conduits individuellement, dans ces mêmes loges $(1,5$ $\mathrm{m} \times 0,8 \mathrm{~m} \times 0,6 \mathrm{~m}$ ), jusqu'à 8 semaines d'âge. Chaque loge était tapissée d'une couche de copeaux de bois non traités de $5 \mathrm{~cm}$ d'épaisseur renouvelée tous les 7 jours pour éviter l'accumulation des fèces et des urines.

\section{Conduite de l'essai}

Lors de la conduite de l'essai, les femelles ont été réparties dans un dispositif complètement randomisé avec 25 répétitions par traitement ou lot. Au début de l'expérience, les animaux ont été mis en croisement (4 mâles pour 25 femelles) pendant 31 jours et les mâles ont ensuite été isolés. La formulation des rations, a été précédée par l'analyse bromatologique de celles-ci selon les méthodes A.O.A.C (1990). La teneur en protéines des différentes rations de gestation (RG18, RG20 et RG22) était respectivement de $18 \%, 20 \%$ et $22 \%$. Chaque animal des différents lots recevait quotidiennement 31,42 g MS de l'aliment conditionné sous forme de granulés de $4 \mathrm{~mm}$ de diamètre et de l'eau vitaminée à volonté (comprimé de $240 \mathrm{mg}$ de vitamine $\mathrm{C}$ dans 1,5 litres d'eau). Les cobayes ont été identifiés à l'aide des boucles métalliques numérotées. A l'exception du P. purpureum, tous les autres ingrédients entrant dans la fabrication de la ration ont été achetés chez les revendeurs de sous-produits agricoles de la ville de Dschang. 
Cette graminée, récoltée à la FAR, était hachée, séchée, broyée et ensuite était incorporée dans les différentes rations. Un échantillon de $100 \mathrm{~g}$ de chaque ration expérimentale a été prélevée et séchée à $60^{\circ} \mathrm{C}$ jusqu'à poids constant dans une étuve ventilée de marque Gallemkamp. Les échantillons ont été broyés aux mailles de $1 \mathrm{~mm}$ et conservés dans des sachets en plastique en vue des différentes analyses bromatologiques.

Pour l'essai de la reproduction, trois rations expérimentales (Tableau 1) isoénergétiques (énergie digestible $=2800 \mathrm{Kcal} /$ $\mathrm{kgMS}$ ) ont été formulées, contenant respectivement $18 \%, 20 \%$ et $22 \%$ de protéines brutes :

- RG18 = Ration avec $18 \%$ de protéines (Lot1, $\mathrm{n}=25$ )

- RG20 = Ration avec $20 \%$ de protéines (Lot2, $\mathrm{n}=25$ )

- RG22 = Ration avec $22 \%$ de protéines (Lot $3, \mathrm{n}=25$ )

Pour l'essai de la croissance, trois rations expérimentales (Tableau 2) isoénergétiques (énergie digestible $=2800 \mathrm{Kcal} /$ $\mathrm{kgMS}$ ) ont été formulées, contenant respectivement $14 \%, 16 \%$ et $18 \%$ de protéines brutes. :

- $\mathbf{R C 1 4}=$ Ration avec $14 \%$ de protéines (Lot1, $\mathrm{n}=18$ )

- $\mathbf{R C 1 6}=$ Ration avec $16 \%$ de protéines (Lot2, $\mathrm{n}=18$ )

- $\mathbf{R C 1 8}=$ Ration avec $18 \%$ de protéines (Lot3, $\mathrm{n}=18$ )

\section{Performances de reproduction}

Pour l'évaluation des performances de reproduction, au début de l'essai, l'effectif des femelles mises en reproduction a été enregistré ainsi que leurs poids. Au cours de la gestation, l'effectif de femelles ayant avorté a été noté. L'effectif des femelles ayant mis bas ainsi que la date de mise bas ont été par la suite enregistrés. A la naissance, le nombre de cochonnets mort-nés et nés vivants ont été notés. Ensuite, le nombre de cochonnets morts avant le sevrage et le nombre de petits sevrés ont été également enregistrés. Par ailleurs, après la mise bas, les mères et les cochonnets ont été pesés tous les 7 jours jusqu'au sevrage des petits.

\section{Performances de croissance}

Concernant l'évaluation des performances de croissance, les refus des différentes rations étaient collectés tous les matins et pesés à l'aide d'une balance digitale de capacité $5 \mathrm{~kg}$ et de sensibilité $1 \mathrm{~g}$. Pour l'évaluation de la croissance pondérale, les poids des mères étaient pris à la mise bas, ensuite, tous les 7 jours jusqu'au sevrage (21 jours). Les petits ont également été pesés à la naissance et de façon hebdomadaire jusqu'à 8 semaines d'âge.

\section{Analyses statistiques}

Les données sur la reproduction et la croissance des femelles reproductrices ont été soumises à une analyse de la variance à un facteur (ration) suivant le modèle linéaire général (MLG). Par ailleurs, les données sur la croissance des jeunes ont été soumises à l'analyse de la variance à 2 facteurs (ration et sexe) suivant le MLG. Lorsque les différences étaient significatives entre les traitements, les moyennes étaient séparées par le test de Duncan au seuil de $5 \%$ (Steel et Torrie 1980) et le test « $\mathrm{t} »$ de Student a été utilisé pour faire la comparaison entre les sexes. 
Tableau 1: Formule et composition chimique des rations expérimentales pour la période de gestation.

\begin{tabular}{|c|c|c|c|}
\hline \multirow{2}{*}{ Ingrédients } & \multicolumn{3}{|c|}{ Proportion des ingrédients (\%) } \\
\hline & RG1 (18\%) & RG2 (20 \%) & RG3 (22 \%) \\
\hline Maïs & 18 & 16,5 & 14 \\
\hline Résidus de semoule de manioc & 8 & 8 & 8 \\
\hline Son de blé & 32,5 & 32 & 29 \\
\hline Tourteau de coton & 5 & 7 & 6,5 \\
\hline Tourteau de soja (49\%) & 4 & 7,5 & 13,5 \\
\hline Farine de poisson $(60 \%)$ & 9 & 9 & 9 \\
\hline Farine de coquillage & 2 & 2 & 2 \\
\hline Sel iode & 1 & 1 & 1 \\
\hline Pennisetum purpureum & 19,5 & 16 & 16 \\
\hline Prémix $2 \%{ }^{*}$ & 1 & 1 & 1 \\
\hline Total & 100 & 100 & 100 \\
\hline \multicolumn{4}{|l|}{ Composition chimique } \\
\hline MS (\%) & 91,28 & 91,74 & 92,45 \\
\hline MO (\%MS) & 84,25 & 94,12 & 82,86 \\
\hline Protéine brute $(\% \mathrm{MS})$ & 18,11 & 20,06 & 22,07 \\
\hline Cellulose brute (\%MS) & 11,74 & 10,87 & 10,60 \\
\hline Cendres (\%MS) & 15,75 & 15,88 & 17,14 \\
\hline $\mathrm{Ca}$ & 1,49 & 1,49 & 1,49 \\
\hline $\mathrm{p}$ & 0,79 & 0,81 & 0,79 \\
\hline $\mathrm{Ca} / \mathrm{P}$ & 1,89 & 1,84 & 1,89 \\
\hline ED (Kcal/KgMS) & 2804,05 & 2804,13 & 2806,36 \\
\hline $\mathrm{ED} / \mathrm{PB}$ & 154,83 & 139,79 & 127,16 \\
\hline
\end{tabular}


Tableau 2: Formule et composition chimique des rations expérimentales pour la période de croissance.

\begin{tabular}{lccc}
\hline \multirow{2}{*}{ Ingrédients } & \multicolumn{3}{c}{ Proportion des ingrédients (\%) } \\
\cline { 2 - 4 } & RC1 (14\%) & RC2 (16\%) & RC3 (18\%) \\
\hline Maïs & 23 & 22 & 18 \\
Résidus de semoule de manioc & 8 & 7,5 & 8 \\
Son de blé & 34,5 & 34 & 32 \\
Tourteau de coton & 0 & 2 & 5 \\
Tourteau de soja (49\%) & 0 & 1 & 4 \\
Farine de poisson (60\%) & 7 & 9 & 9 \\
Farine de coquillage & 2 & 2 & 2 \\
Sel iode & 1,5 & 1,5 & 1 \\
Pennisetum purpureum & 23 & 20 & 20 \\
Prémix 2\% & 1 & 1 & 1 \\
Total & 100 & 100 & 100 \\
\hline Composition chimique & & & \\
\hline MS (\%) & 89,77 & 93,45 & 90,42 \\
MO (\%MS) & 85,35 & 84,67 & 83,23 \\
Protéine brute (\%MS) & 14,02 & 16,06 & 18,09 \\
Cellulose brute (\%MS) & 12,30 & 11,55 & 11,86 \\
Cendres (\%MS) & 14,65 & 15,33 & 16,77 \\
Ca & 1,35 & 1,48 & 1,49 \\
p & 0,72 & 0,79 & 0,79 \\
Ca/P & 1,88 & 1,87 & 1,89 \\
ED (Kcal/KgMS) & 2805,94 & 2806,96 & 2808,32 \\
ED/PB & 200,14 & 174,78 & 155,24 \\
\hline Vi A 00000
\end{tabular}

Vit A : 3000000 UI, Vit D $3: 600000$ UI, Vit E :4 $000 \mathrm{mg}$, Vit K : $500 \mathrm{mg}$, Vit B1 : $200 \mathrm{mg}$, VitB2 : $1000 \mathrm{mg}$, Vit B6 : $400 \mathrm{mg}$, Vit B12 :4 mg, Fer : $8000 \mathrm{mg}, \mathrm{Cu}: 2000 \mathrm{mg}, \mathrm{Zn}: 10000 \mathrm{mg}$, Se : $20 \mathrm{mg}, \mathrm{Mn}: 14000 \mathrm{mg}$, Méthionine : 200000 $\mathrm{mg}$, Lysine : $78000 \mathrm{mg}$. RC1 ; RC2 ; RC3 : 14\% ; 16\%; 18\% de taux de protéines alimentaires respectivement.

\section{RESULTATS}

\section{Effets du niveau de protéines alimentaires} sur les performances de reproduction

Le taux de fertilité a augmenté avec le niveau de protéines alimentaires de la ration. En effet, la ration contenant $22 \%$ de $\mathrm{PB}$ (RG22) a permis d'obtenir le taux le plus élevé (96\%) et la ration contenant $18 \%$ de PB (RG18) le taux le plus faible (28\%) (Tableau $3)$.

Les tailles de la portée étaient de 1,71 ; 1,80 et 1,92 respectivement pour les animaux des lots PB18; PB20 et PB22; la valeur la plus élevée a été enregistrée chez les femelles du lot PB22; cependant, aucune différence significative $(\mathrm{p}>0,05)$ n'a été observée entre ces valeurs.

Les poids moyens à la naissance ont augmenté avec le niveau de protéines alimentaires. Ces poids étaient de $61,14 \pm 16,19 ; 81,87 \pm 5,11$ et $98,36 \pm 18,47$ respectivement pour les cobayes des lots PB18; PB20 et PB22. Les poids des jeunes issus des femelles du lot PB22 ont été significativement $(\mathrm{p}<0,05)$ plus élevés que ceux des jeunes issus des femelles des lots PB18 et PB20; et ceux des jeunes issus des femelles du lot PB20 ont été significativement $(p<0,05)$ plus élevés que ceux des lots des 
jeunes issus des femelles du lot PB18 (18\% de $\mathrm{PB})$.

Le taux de fécondité, de fécondité nette, de viabilité à la naissance et au sevrage ont augmenté avec le niveau de protéines alimentaires. Les valeurs les plus faibles $(48 \%, \quad 28 \%, \quad 58,33 \%$ et $71,43 \%$ respectivement) ayant été obtenues chez les reproductrices du lot PB18 et les plus élevées $(184 \%, \quad 184 \%, \quad 100 \%$ et $97,83 \%$ respectivement) chez celles du lot PB22.

Par ailleurs, de la mise-bas au sevrage, le poids moyen hebdomadaire de toutes les femelles allaitantes a chuté dans tous les lots (Figure 1). Néanmoins, le poids des femelles du lot PB22 est resté supérieur à ceux des femelles des lots PB18 et PB20 durant toute cette période. Aucune différence significative $(\mathrm{P}>0,05)$ n'a cependant été observée entre les différentes valeurs, bien que les femelles allaitantes du lot PB22 aient présenté la chute de poids la plus faible.

Effet du niveau de protéines alimentaires sur les gains de poids et l'indice de consommation des cochonnets entre la $3^{\text {ème }}$ et la $8^{\text {ème }}$ semaine d'âge

Le poids le plus élevé $(221,08 \mathrm{~g})$ à la $8^{\text {ème }}$ semaine a été obtenu chez les animaux du lot PB16 (RC16) et le plus faible $(182,24 \mathrm{~g})$ chez ceux du lot PB14 (RC14) (Tableau 4). Les poids moyens des animaux des lots PB16 et PB18, sont restés comparables $(\mathrm{p}>0,05)$ mais significativement $(p<0,05)$ plus élevés que celui obtenu chez les cobayes du lot PB14. Par ailleurs, le gain total et le GMQ les plus élevés, respectivement $56,70 \mathrm{~g}$ et $2,02 \mathrm{~g} / \mathrm{j}$, ont été enregistrés chez les cobayes du lot PB16 et les plus faibles, respectivement 16,75 $\mathrm{g}$ et $0,60 \mathrm{~g} / \mathrm{j}$, chez ceux du lot PB14. L'analyse statistique a révélé que le gain total et le GMQ des animaux soumis à la ration contenant $16 \%$ de $\mathrm{PB}$ ont été significativement $(\mathrm{p}<0,05)$ plus élevés que ceux des cobayes alimentés aux rations contenant $18 \%$ et $14 \%$ de PB. Par ailleurs, ceux des animaux du lot PB18 ont été significativement $(\mathrm{p}<0,05)$ supérieurs à ceux des animaux du lot PB14. L'indice de consommation le plus faible $(2,12)$ a été enregistré chez les animaux alimentés à la ration contenant $16 \%$ de $\mathrm{PB}$ et le plus élevé $(5,20)$ chez ceux du lot PB14. Par ailleurs, de la $3^{\text {ème }}$ à la $8^{\text {ème }}$ semaine, les rations ayant des taux de $14 \%$ et $16 \%$ de $\mathrm{PB}$, respectivement $\mathrm{RC} 14$ et $\mathrm{RC} 16$, ont permis aux mâles d'avoir les gains totaux et les GMQ significativement $(\mathrm{P}<0,05)$ meilleurs comparés à ceux des femelles.

Tableau 3 : Les performances moyennes de reproduction chez les femelles en fonction des rations.

\begin{tabular}{lccc}
\hline \multirow{2}{*}{ Paramètres } & \multicolumn{3}{c}{ Traitements } \\
\cline { 2 - 4 } & RG1 (18\%) & RG2 (20\%) & RG3 (22\%) \\
\hline Taux de fertilité (\%) & 28 & 40 & 96 \\
Taille de la portée & 1,71 & 1,80 & 1,92 \\
Poids moyen à la naissance & $61,14^{\mathrm{c}}$ & $81,87^{\mathrm{b}}$ & $98,36^{\mathrm{a}}$ \\
Taux de fécondité (\%) & 48 & 72 & 184 \\
Taux de fécondité nette (\%) & 28 & 60 & 184 \\
Taux de viabilité à la naissance (\%) & 58,33 & 83,33 & 100 \\
Taux de viabilité au sevrage (\%) & 71,43 & 93,33 & 97,83 \\
Poids moyen au sevrage & $164,17 \pm 9,08$ & $164,22 \pm 8,68$ & $164,28 \pm 9,22$ \\
\hline
\end{tabular}

a, b et c : les moyennes portant les mêmes lettres en exposant minuscules sur la même ligne sont statistiquement identiques ; RG1, RG2, RG3: $18 \% ; 20 \% ; 22 \%$ de taux de protéines alimentaires respectivement. 
Tableau 4 : Poids moyens à la 8è semaine, gains totaux, GMQ et indice de consommation des cobayes en fonction des traitements.

\begin{tabular}{|c|c|c|c|c|c|}
\hline \multirow{2}{*}{ Caractéristiques } & \multicolumn{5}{|c|}{ Traitements } \\
\hline & RC1 (14\%) & RC2 (16\%) & RC3 (18\%) & ESM & Prob. \\
\hline \multicolumn{6}{|l|}{ Poids au sevrage (g) } \\
\hline$\pi$ & $168,04^{\mathrm{aA}}$ & $168,11^{\mathrm{aA}}$ & $168,04^{\mathrm{aA}}$ & 1,53 & 0,999 \\
\hline$q$ & $160,33^{\mathrm{aB}}$ & $160,36^{\mathrm{aB}}$ & $160,58^{\mathrm{aB}}$ & 1,57 & 0,998 \\
\hline$j o$ & $164,17^{\mathrm{a}}$ & $164,22^{\mathrm{a}}$ & $164,28^{\mathrm{a}}$ & 1,20 & 0,999 \\
\hline \multicolumn{6}{|l|}{$\begin{array}{l}\begin{array}{l}\text { Poids à la } \\
\text { semaine }(\mathrm{g})\end{array} \\
\text { sème }\end{array}$} \\
\hline$\lambda$ & $191,04^{\mathrm{cA}}$ & $228,31^{\mathrm{aA}}$ & $216,44^{\mathrm{bA}}$ & 3,47 & 0,000 \\
\hline 우 & $173,43^{\mathrm{bB}}$ & $213,84^{\mathrm{aB}}$ & $211,98^{\mathrm{aA}}$ & 4,09 & 0,000 \\
\hline$j o$ & $182,24^{\mathrm{b}}$ & $221,08^{\mathrm{a}}$ & $214,21^{\mathrm{a}}$ & 2,76 & 0,000 \\
\hline \multicolumn{6}{|l|}{ Gains totaux $(\mathrm{GT})(\mathrm{g})$} \\
\hline$\hat{\sigma}$ & $22,00^{\mathrm{cA}}$ & $60,20^{\mathrm{aA}}$ & $48,40^{\mathrm{bA}}$ & 3,15 & 0,000 \\
\hline 우 & $11,14^{\mathrm{bB}}$ & $52,72^{\mathrm{aB}}$ & $51,40^{\mathrm{aA}}$ & 3,90 & 0,000 \\
\hline j우 & $16,75^{\mathrm{c}}$ & $56,70^{\mathrm{a}}$ & $49,90^{\mathrm{b}}$ & 2,48 & 0,000 \\
\hline \multicolumn{6}{|l|}{ GMQ (g/j) } \\
\hline$\pi$ & $0,78^{\mathrm{cA}}$ & $2,15^{\mathrm{aA}}$ & $1,73^{\mathrm{bA}}$ & 0,11 & 0,000 \\
\hline q & $0,40^{\mathrm{bB}}$ & $1,88^{\mathrm{aB}}$ & $1,84^{\mathrm{aA}}$ & 0,14 & 0,000 \\
\hline jo우 & $0,60^{\mathrm{c}}$ & $2,02^{\mathrm{a}}$ & $1,78^{\mathrm{b}}$ & 0,09 & 0,000 \\
\hline \multicolumn{6}{|l|}{ IC } \\
\hline$\hat{\sigma}$ & 3,50 & 1,67 & 2,31 & l & l \\
\hline q & 7,09 & 2,63 & 3,33 & I & I \\
\hline$j o$ & 5,20 & 2,12 & 2,82 & 1 & I \\
\hline
\end{tabular}

$\mathrm{a}, \mathrm{b}$ et $\mathrm{c}$ : Les moyennes portant les mêmes lettres minuscules sur la même ligne ne sont pas significativement différentes au seuil de 5\%; A, B : Les moyennes portant les mêmes lettres majuscules sur la même colonne sont statistiquement identiques au seuil de 5\%, RC1, RC2, RC3: 14\%; $16 \% ; 18 \%$ de taux de protéines alimentaires respectivement ; GT : Gains totaux ; GMQ : Gain moyen quotidien; IC= Indice de consommation; $\widehat{\delta}=$ mâle ; $q=$ femelle ESM : Erreur Standard sur la Moyenne; Prob : Probabilité 


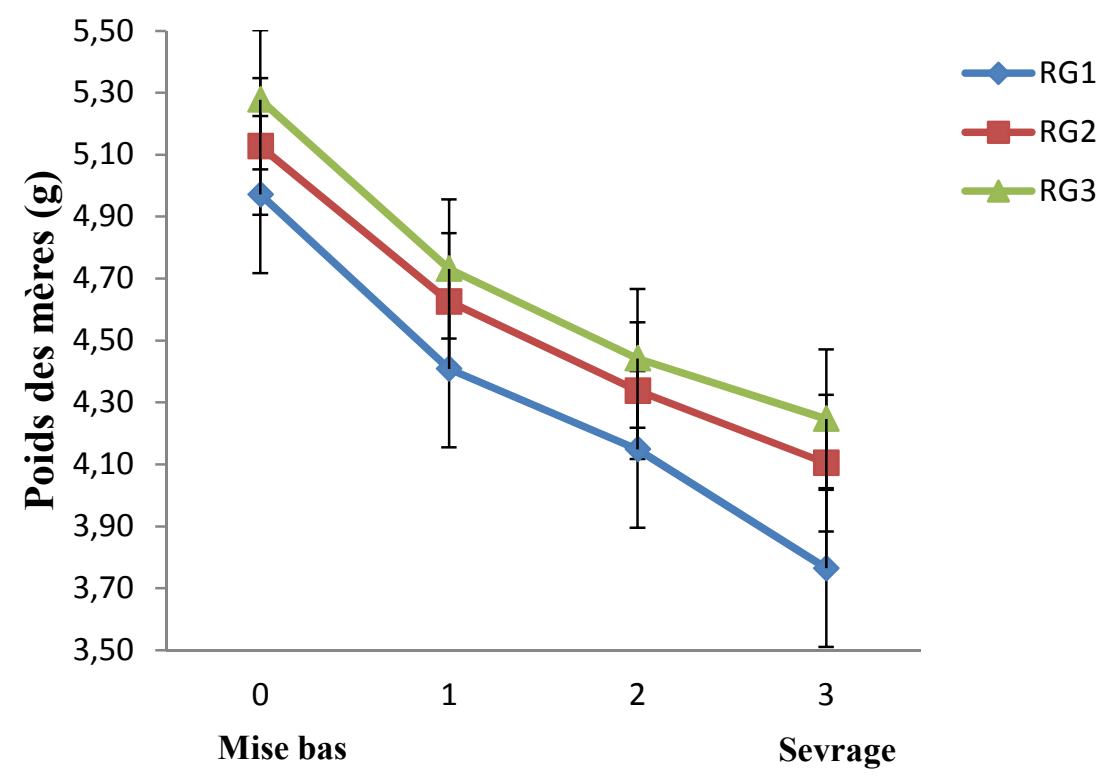

Durée de lactation (semaines)

RG1, RG2, RG3: $18 \% ; 20 \% ; 22 \%$ de taux de protéines alimentaires respectivement.

Figure 1 : Evolution des poids des mères allaitantes de la naissance au sevrage des petits.

\section{DISCUSSION}

Le taux de fertilité a augmenté avec le niveau croissant d'incorporation de protéines brutes dans la ration des reproductrices. Le taux de fertilité le plus élevé obtenu dans cette étude $(96 \%)$ est supérieur à ceux rapportés par Noumbissi (2016) (93,33\%) chez des cobayes nourris à la ration contenant $19,38 \%$ de $\mathrm{PB}$ et par Mweugang et al. (2016) (93,33\%) chez des cobayes recevant $18,43 \%$ de PB dans leur ration et par Kouakou et al. (2012) (75\%) qui ont nourri les cobayes avec la ration contenant $9,60 \%$ de PB. Le taux de fécondité a également augmenté avec le niveau croissant d'incorporation de protéines brutes dans la ration des reproductrices. Les femelles recevant $22 \%$ de $\mathrm{PB}$ dans leur ration ont présenté le taux le plus élevé (184\%). Ce taux a été comparable à ceux obtenus par Kouakou et al. (2012) (187,5\%) chez les cobayes femelles alimentées à la ration contenant
$22,60 \%$ de PB; par Noumbissi (2016) (180\%) chez des cobayes soumis à la ration contenant $19,38 \%$ de PB. Par ailleurs, ce taux est supérieur aux 128,50\% et inférieur aux $206,67 \%$ rapporté par Mweugang et al. (2016), chez des cobayes ayant respectivement $17,87 \%$ et $19,93 \%$ de $\mathrm{PB}$ dans leur ration.

La taille de la portée, dans cet essai, n'a pas été influencée par l'alimentation. Le même constat a été fait par Mweugang et al. (2016), par Miégoué (2016) et par Noumbissi (2016). Ceci pourrait s'expliquer par le rang de mise bas des femelles reproductrices; en effet, celles-ci étaient des primipares et selon Tchoumboué et al. (2001) et Noumbissi (2016) la taille de la portée est liée au numéro de mise-bas des reproductrices et la première portée est toujours plus faible chez les primipares. La taille de la portée la plus élevée $(1,92)$ a été obtenue chez les femelles recevant 
$22 \%$ de PB dans leur ration. Cette valeur est comparable à celles rapportées par Noumbissi (2016) $(2,09)$ chez des cobayes soumis à la ration contenant $29,88 \%$ de $\mathrm{PB}$ et par Kouakou et al. $(2012)(1,80)$ chez des cobayes femelles soumises à la ration contenant $22,60 \%$ de PB.

Les viabilités à la naissance et au sevrage ont été plus élevées chez les femelles recevant le taux de PB alimentaire le plus élevé (22\%). Ce constat pourrait être lié entre autre à l'alimentation. En effet, chez les jeunes cobayes, l'alimentation est un facteur de viabilité tant à la naissance qu'au sevrage (Tchoumboué et al., 2001). Le poids à la naissance est un paramètre déterminant pour la survie des jeunes cobayes (Niba et al., 2008). Tchoumboué et al., (2001) et Noumbissi (2016) ont remarqué que la viabilité augmentait avec le niveau de protéine dans la ration. Le taux de viabilité à la naissance le plus élevé (100\%), obtenu dans la présente étude, est comparable à ceux rapportés par Miégoué (2016) (100\%) chez des cobayes soumis aux rations contenant $16,26 \%$ et $16,07 \%$ de PB et par Mweugang et al. (2016) (100\%) chez des femelles contenant $17,87 \%$ ou $19,92 \%$ de PB dans leur ration. Par ailleurs, ce taux est supérieur à ceux rapportés par Noumbissi (2016) (96,30\%) chez des cobayes alimentés à la ration contenant $19,38 \%$ de PB et par Miégoué (2016) $(91,67 \%)$ chez des cobayes nourris à la ration contenant $16,36 \%$ de PB. Le taux de viabilité au sevrage ou taux de sevrage le plus élevé $(97,83 \%)$ dans cette étude, obtenu chez les petits issus des femelles alimentées à la ration contenant $22 \%$ de $\mathrm{PB}$, est supérieur à ceux rapportés par Noumbissi (2016) (88,46\%) chez des cobayes nourris à la ration contenant $19,38 \%$ de PB ; par Mweugang et al. (2016) $(95,04 \%)$ chez des femelles soumises à la ration contenant $18,43 \%$ de PB ; par Miégoué (2016) $(90,91 \%)$ chez des cobayes nourris à la ration contenant $16,36 \%$ de $\mathrm{PB}$.
De la mise-bas au sevrage, le poids moyen hebdomadaire de toutes les femelles allaitantes a baissé. Ces résultats sont en accord avec les observations de Pamo et al. (2005); de Noumbissi (2016); Miégoué et al. (2016) ; Mweugang et al. (2016). En effet, au cours de cette période, les mères dépensent beaucoup d'énergie pour la production du lait (Michel et Bonnet, 2012). Pour assurer une production laitière satisfaisante, elles sont obligées de mobiliser leurs réserves corporelles, ce qui expliquerait la perte de poids observée. Selon ces mêmes auteurs, cette perte est plus accentuée chez les femelles non complémentées. La perte de poids la moins élevée dans cette étude a été observée chez les allaitantes du lot recevant $22 \%$ de PB dans leur ration. Ceci serait dû à la quantité de protéines alimentaires plus élevée dans cette ration par rapport aux autres.

Le poids moyen au sevrage $(164,31 \mathrm{~g})$ obtenu dans la présente étude, chez les petits issus des femelles du lot PB22, est comparable à ceux enregistrés par Miégoué (2016) $(166,09 \mathrm{~g})$ chez des cobayes soumis à la ration contenant $16,23 \%$ de $\mathrm{PB}$ et par Noumbissi (2016) (162,64 g) chez ceux soumis à la ration contenant 19,38\% de PB, mais supérieur à celui rapporté par Miégoué (2016), avec la ration contenant $16,36 \%$ de PB (159,60 g). Au sevrage, les mâles étaient plus lourds que les femelles. Ceci confirme les observations faites par Noumbissi (2016) et par Niba et al. (2004a) selon lesquelles, les mâles croissent plus vite que les femelles.

Dans la présente étude, le gain total et le GMQ les plus élevés, respectivement 56,70 $\mathrm{g}$ et $2,02 \mathrm{~g} / \mathrm{j}$ ont été obtenus chez les cobayes du lot PB16. Ce GMQ est supérieur à celui rapporté par Kouakou et al. (2010) $(0,9$ g / j) chez les cobayes alimentés à la ration contenant $10,20 \%$ de $\mathrm{PB}$, mais comparable à ceux obtenus par Noumbissi (2016), 2,49 g / j et $1,93 \mathrm{~g} / \mathrm{j}$, respectivement chez des cobayes soumis aux rations contenant $24,63 \%$ et 
$29,88 \%$ de PB. Par ailleurs, il est inférieur à celui obtenu par Miégoué (2016) (3,58 g / j) chez des cobayes alimentés à la ration contenant $16,36 \%$ de PB. L'indice de consommation le plus faible dans la présente étude $(2,12)$ a été obtenu chez les cobayes recevant la ration contenant $16 \%$ de $\mathrm{PB}$. Cette valeur est inférieure à celles rapportées par Niba et al. (2004a), 8,79; 11,88 et 18,88 , respectivement chez des cobayes alimentés aux rations contenant $28,10 \% ; 26,80 \%$ et $28,80 \%$ de PB. Les fortes valeurs d'indice obtenues par Niba et al. (2004a) pourraient se justifier par une valeur nutritive plus faible des aliments, due particulièrement à la présence de facteurs antinutritionnels dans ces rations. En effet, les travaux de Noumbissi (2016), menés sur les cobayes nourris à l'aide de rations complémentées avec des feuilles fraîches de $T$. diversifolia, une plante reconnue pour sa forte concentration en facteurs antinutritionnels, ont révélé une chute de poids chez ces animaux liée vraisemblablement à la présence de ces facteurs dans lesdits régimes alimentaires.

\section{Conclusion}

Il ressort de cette étude que le poids à la naissance des cochonnets, les taux de fertilité, de fécondité, de fécondité nette, de viabilité à la naissance et de viabilité au sevrage, les plus élevés, ont été enregistrés chez les femelles recevant la ration contenant $22 \%$ de $\mathrm{PB}$ (RG22). Du sevrage à 8 semaines d'âge, les meilleurs gains totaux, gains moyens quotidiens et indices de consommation ont été obtenus chez les cobayes nourris à la ration contenant $16 \%$ de PB (RC16). Ces paramètres sont plus intéressants chez les mâles comparés à ceux des femelles. Au regard de ces résultats, les rations contenant $22 \%$ et $16 \%$ de $\mathrm{PB}$, conviennent mieux aux cobayes à l'Ouest Cameroun en gestation (RG22) et en croissance ( $\mathrm{RC16}$ ), respectivement. Il serait aussi souhaitable d'évaluer l'effet du taux de $16 \%$ de PB sur les performances de croissance et des caractéristiques de la carcasse chez les cobayes de 15 à 22 semaines d'âges.

\section{CONFLITS D'INTERETS}

Les auteurs déclarent qu'ils n'ont aucun conflit d'intérêt.

\section{CONTRIBUTIONS DES AUTEURS}

GTZ a conçu, a mis en place le dispositif expérimental, a réalisé des essais, a collecté des données et a rédigé le manuscrit; FT a participé à la mise en place du dispositif expérimental, à la conduite des essais et à la lecture critique du manuscrit; EM a participé à l'assistance technique et scientifique du travail et à la lecture critique du manuscrit; MNBN, AVM, FENM ont participé à la révision du manuscrit et à l'assistance scientifique dans la réalisation de ce travail; $\mathrm{BB}$ et EPT ont coordonné et orienté la réalisation technique et scientifique du travail. Tous les auteurs ont lu et approuvé cette version du travail.

\section{REFERENCES}

Awono Bessa C, Laroche Dupraz C, Grongnet J-F, Vermersch D, Havard M, Lhuissier A. 2005. Déterminants de la consommation urbaine de poulet de chair au Cameroun: cas de la ville de Yaoundé. Agriculture et développement urbain en Afrique de l'Ouest et du Centre, tome 1: Gouvernance et approvisionnement des villes, actes des ateliers de Yaoundé 30/octobre - 3 novembre 2005, 2008, IRAD-CIRAD, p. 193-202.

Bindelle J, Picron P. 2013. Le cobaye, un petit herbivore facile à nourrir dans des petites parcelles. Troupeaux et Cultures des Tropiques : Spécial Elevage de Rongeurs. CAVTK : Kinshasa, RDC. 110. 
Boucher S. 1995. Alimentation des lagomorphes et rongeurs de compagnie : quelques données de base. In Congrès Annuel : "Conduite Diagnostique et Thérapeutique : Actualités ». CNVSPA: Paris ; 151-160.

Fantodji A, Soro D, Mensah G A. 2004. Reproduction et croissance des aulacodes (Thryonomys swinderianus) élevés en captivité étroite en Côte d'Ivoire. Sciences et Nature, 1: 25-33.

Fonteh FA, Niba AT, Kudi AC, Tchoumboue J, Awah-Ndukum J. 2005. Influence of weaning age on the growth performance and survival of weaned guinea pigs. Livestock Research for Rural Development. 17, http://www.cipav.org. co/lrrd//rrd17/12/font17133.htm

Fotsa JG, Poné DK, Manjeli Y, Mafeni Mase J. 2007. Etude des systèmes d'élevage et description phénotypique des poules locales (Gallus gallus) en milieu rural de la zone forestière du Cameroun. Cameroon Journal of Agricultural Science, 3: 40-47.

Kondombo SR, Kwakkel, RP, Nianogo AJ, Slingerland M. 2003. Effects of local feedstuff supplementation on zootechnic performances and nutritional status of village chickens during the end of the rainy season in Burkina Faso. Revue d'Elev. Méd. Vét. Pays Trop., 56: 3-4

Kouakou N'GDV, Thys E, Assidjo EN, Grongnet JF. 2010. Ingestion et digestibilité in vivo du Panicum maximum associé à trois compléments: tourteau de Jatropha curcas, tourteau de coton (Gossypium hirsutum) et Euphorbia heterophylla chez le cobaye (Cavia porcellus L.). Tropicultura, 28(3) : 173-177.

Kouakou N'GDV, Thys E, Danho M, Assidjo EN, Grongnet JF. 2012. Effet de Panicum maximum sur la productivité des femelles primipares durant le cycle de reproduction chez le cobaye (Cavia porcellus L.). Tropicultura, 30(1): 24-36. Michel CL, Bonnet X. 2012. Influence of body condition on reproductive output in the guinea pig. J. Exp. Zool., 317: 24 31. Doi: $10.1002 /$ jez.714

Miégoué E. 2016. Trois légumineuses fourragères (Arachis glabrata, Calliandra calothyrsus ou Desmodium intortum) comme source de protéines associées à deux graminées (Pennisetum purpureum et Panicum maximum) dans l'alimentation des cochons d'inde ((Cavia porcellus L.). Thèse de Doctorat $(\mathrm{PhD})$ en Biotechnologie et Productions Animales. Université de Dschang, 158p.

Miégoué E, Tendonkeng F, Lemoufouet J, Noumbissi MNB, Mweugang NN, Zougou TG, Nkouadjio MB, Boukila B, Pamo TE. 2016. Croissance pré-sevrage des cobayes nourris au Panicum maximum supplémentés avec une ration contenant Arachis glabrata, Calliandra calothyrsus ou Desmodium intortum. Int. J. Biol. Chem. Sci., 10(1): 313-325. Doi :

http://dx.doi.org/10.4314/ijbcs.v10i1.24

Mweugang NN. 2016. Utilisation des feuilles de manioc (Manihot esculenta Crantz) comme source alternative de protéines sur les performances de production du cobaye (Cavia porcellus L.) et la composition chimique de sa viande. Thèse de Doctorat (PhD) en Biotechnologie et Productions Animales. Université de Dschang. 166p.

Mweugang NN, Tendonkeng F, Miégoué E, Matumuini FEN, Zougou TG, Fonteh FA, Boukila B, Pamo TE. 2016. Effets de l'inclusion de feuilles de manioc (Manihot esculenta Crantz) dans la ration sur les performances de reproduction du cobaye (Cavia porcellus L.) local camerounais. Int. J. Biol. Chem. 
Sci., 10(1): 269-280. Doi : http://dx.doi.org/10.4314/ijbcs.v10i1.21

Niba AT, Djoukam J, Teguia A, Kudi AC, Loe JO. 2004a. Influence of level of cotton seed cake in the diet on the feed intake, growth performance and carcass characteristics of guinea pigs in Cameroon. Tropicultura. 22(1): 32-39.

Niba AT, Kudi AC, Tchoumboue J, Zoli PA, Fonteh FA, Komtangi MC. 2004b. Influence of birth weight and litter size on the preweaning growth performance and survival of guinea pigs (Cavia porcellus L.). Journal of the Cameroon Academy of Sciences, 4: 19-25.

Niba AT, Kudi AC, Fonteh F, Tchoumboue J. 2008. Influence of birthweight and litter size on the growth performance of guinea pigs under intensive management. Sciences Agronomiques et Développment, 4(1): 13-20.

Niba AT, Meutchieye F, Fon D, Laisin AG, Taboh H, Njakoi H, Bela T A, Maass B L, Djikeng A, Manjeli Y. 2012. Current situation of cavy production in Cameroon: Challenges and opportunities. Livestock Research for Rural Development, 24, http://www.lrrd.org/lrrd24/11/niba24194. h.

Nkidiaka O. 2004. Les pratiques d'élevage en milieu urbain et péri urbain de la ville de Kinshasa : synthèse générale des enquêtes. Troupeaux et Cultures des Tropiques, 3 : 50-52.

Noumbissi MNB, Tendonkeng F, Zougou TG, Pamo TE 2014. Effet de différents niveaux de supplémentation de feuilles de Tithonia diversifolia (Hemsl) A Gray sur l'ingestion de la digestibilité in vivo de Pennisetum purpureum K. Schum. Chez le cobaye (Cavia porcellus L.). Tropicultur., 32: 3138-146.

Noumbissi MNB, 2016. Evaluation des performances de production des cobayes (Cavia porcellus L.) soumis à différents niveaux de supplémentation avec Tithonia diversifolia. Thèse de Doctorat $(\mathrm{PhD})$ en Biotechnologie et Productions Animales. Université de Dschang. 163p.

Pamo TE, Boukila B, Fonteh FA, Tendonkeng F, Kana JR. 2005. Composition chimique et effet de la supplémentation avec Calliandra calothyrsus et Leucaena leucocephala sur la production laitière et la croissance des chevreaux nains de Guinée. Livestock Research for Rural Development, $17 . \quad \mathrm{http}: / /$ www.lrrd.org/lrrd17/3/tedo17030.htm

Rosse L.1999. Alimentation des rongeurs et lagomorphes de compagnie. Thèse Médecine Vétérinaire. : Alfort: 1999 : 10.

Tchoumboué J, Niba, A T, Kenfack, A. 2001. Comparative study on the influence of supplementation with two legumes (Arachis glabrata Benth. and Desmodium intortum) on the reproductive and growth performance of guinea pigs (Cavia porcellus L.). Bulletin of Animal. Health and Production, 49 : 74-83. 